\title{
"There's only so much you can be pushed": a commentary on the magnification of the maternity staffing crisis by the 2020/21 COVID-19 pandemic
}

\author{
Sarah Cordey $^{1}$, Gill Moncrieff ${ }^{1}$, Joanne Cull ${ }^{1}$, Arni Sarian ${ }^{1}$, Deborah Powney ${ }^{1}$, Dr Carol \\ Kindgon $^{1}$, Dr Claire Feeley ${ }^{1}$, and Professor Soo Downe ${ }^{1}$ \\ ${ }^{1}$ Affiliation not available
}

March 1, 2022

Sarah Cordey RM, School of Community Health and Midwifery, THRIVE Centre, University of Central Lancashire, Preston, UK

Gill Moncrieff RM, Research in Childbirth and Health Group, THRIVE Centre, University of Central Lancashire, Preston, UK

Joanne Cull RM, School of Community Health and Midwifery, THRIVE Centre, University of Central Lancashire, Preston, UK

Arni Sarian, School of Medicine, University of Central Lancashire, Preston, UK

Deborah Powney, MSc, Research in Childbirth and Health Group, THRIVE Centre, University of Central Lancashire, Preston, UK

Dr Carol Kingdon, PhD, Wirral University Teaching Hospital NHS Foundation Trust, Wirral, UK

Dr Claire Feeley, PhD, Research in Childbirth and Health Group, THRIVE Centre, University of Central Lancashire, Preston, UK

Professor Soo Downe PhD, Research in Childbirth and Health Group, THRIVE Centre, University of Central Lancashire, Preston, UK

On behalf of the ASPIRE Covid-19 Team (see Appendix A1)

Corresponding Author: Sarah Cordey

Over the past two decades there has been a developing staffing crisis in British maternity services (1). A 2021 Health and Social Care Committee Expert Panel report described "persistent gaps in all maternity professions" and proposed that "current recruitment initiatives do not consider the serious problem of attrition in a demoralised and overstretched workforce and do not adequately value professional experience and wellbeing" (2). Reduced staff capacity and excessive workload can have a profoundly detrimental impact on safety for women and babies $(3,4)$. The ongoing impact of the COVID-19 pandemic on the National Health Service (NHS) has led to discussion around the long-term effects on staff from acute staffing shortages, the moral distress of being unable to provide the desired level of care, and the heavy emotional labour of being a front-line healthcare worker during a pandemic. (5) 
This commentary explores the impact of these factors on maternity staff during the COVID-19 pandemic and reflects on the implications for the future of maternity services design and delivery. The discussion relates to a thematic analysis (6) and NVivo word frequency analysis of in-depth interviews with 28 maternity staff (20 midwives, seven medical staff and one student midwife), 28 heads of service, and 26 women who gave birth during the pandemic, from seven NHS trusts across England. The interviews took place between November 2020 and October 2021 and were collected as part of the Achieving Safe and Personalised maternity care In Response to Epidemics (ASPIRE COVID-19) study. The overall aim of ASPIRE COVID-19 is to determine what worked to provide safe and personalised care maternity during the pandemic, both to optimise care under usual circumstances, and to improve the response to future crises. The analysis resulted in one overarching theme, and three sub-themes, as illustrated in Figure 1. Supporting quotes are shown in Figure 2 and Table S1.

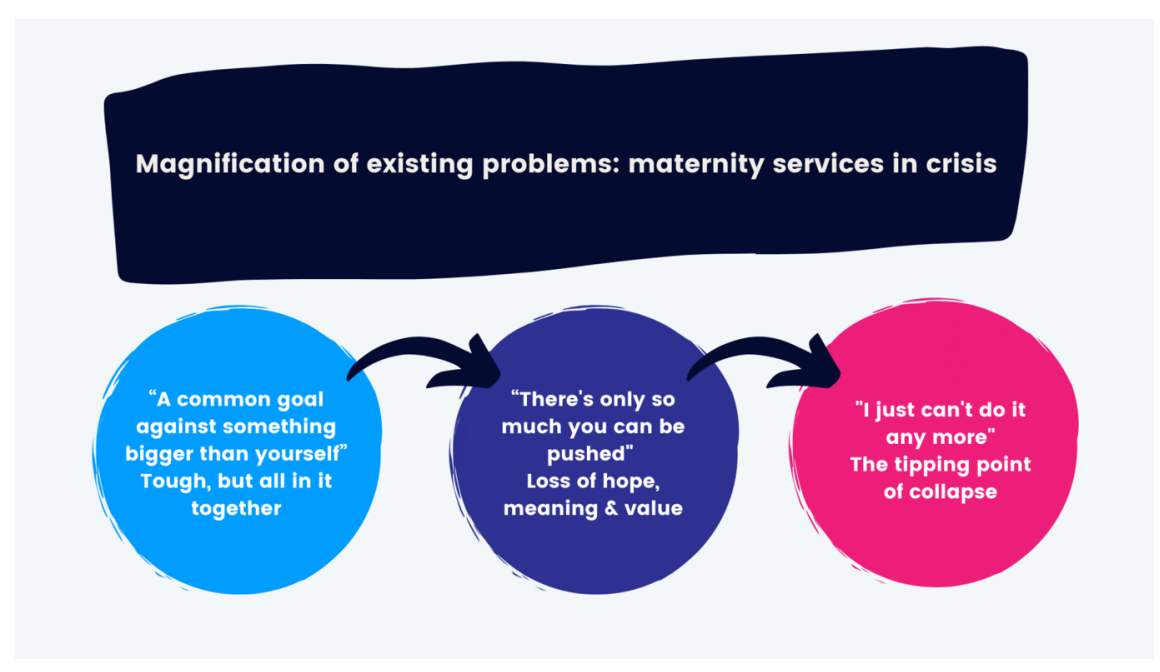

Figure 1: Overarching theme: Magnification of existing problems

\section{Magnification of existing problems: maternity services in crisis}

Our findings showed an emerging chronological narrative of how a sudden influx of staff and resources early in the pandemic, combined with a sense of camaraderie, public support and professional pride, led to an unexpectedly positive work environment. However, these protective factors did not last; the initial boost was quickly followed by warnings of deteriorating morale, compassion fatigue and moral distress. The reasons for this were complex, with interviewees reporting fundamental issues with staff recruitment and retention, deteriorating physical and psychological wellbeing, and unmanageable workloads. A lack of emotional resources and focus on a 'checkbox' style system of care, with overwhelming documentation and supposed safety measures which paradoxically made it harder for staff to be 'with woman', were reported to be at odds with the safe and personalised care that they wanted to provide. The sustained pressures of the pandemic magnified these daily struggles, which led to a loss of meaning and of loss of desire to continue working within maternity.

Respondents reported making a consistent professional effort to maintain safety for service users, sometimes at the expense of their own wellbeing. This may contribute to the fact that around half of the women who were interviewed did not mention staff wellbeing. However, 13 women out of the 26 interviews in our sample indicated that they were very aware of staffing shortages and the way it affected their experience of care, with more reports of dissatisfaction given by those in our sample who birthed later in the pandemic.

\section{"A common goal against something bigger than yourself": Tough, but all in it together}


Staff spoke passionately about the camaraderie and teamwork which characterised the early part of the pandemic. The sudden availability of resources to properly fund infrastructure, such as iPads and longawaited software or basic estate repairs to buildings which had been needed for some time, coupled with fully staffed wards, streamlined services and an eagerness from the public to support the NHS meant that, in their experience, standards of care were maintained and they felt valued. Heads of Service who were interviewed reported a time of enhanced productivity, of freedom to enact changes they had waited years for, largely due to the removal of bureaucratic hurdles and combined efforts of Trust leadership. Despite fears for their own health and worries about 'taking home the virus' to their families, many respondents reported making huge personal sacrifices to be part of something 'bigger than themselves'.

\section{"There's only so much you can be pushed": Loss of hope, meaning \& value}

Freedom to adequately staff and resource the service was short-lived. Some service users interviewed in the later phases of the study expressed dissatisfaction with restrictions in areas such as hospital visiting, access to postnatal care, and changes to their usual midwife. Though they were aware of the pressures faced by staff and were somewhat sympathetic, as social restrictions were lifted, they expected a return to usual services. At the same time, many staff respondents were facing difficulties including illness, a lack of childcare and unmanageable working patterns. National implementation of midwifery Continuity of Carer $(\mathrm{CoC})$ continued as planned, although some respondents reported that rollout of $\mathrm{CoC}$ was paused to maintain the core service due to increasing pressures on the available resources. Heads of Service described complex workforce challenges such as increasing rates of short- and long-term staff sickness (particularly amongst midwives), staff taking early retirement, and vacancies advertised but unfilled. Workload pressures were reported to be intensified, with some participants describing clearly unsafe working practices such as an inability to provide one-to-one care in labour, excessively long working hours, and insufficient staffing ratios. Many staff described the emotional distress of working intensively to maintain standards of care but feeling only able to do the 'bare minimum', focusing on safety but having no emotional resource to offer the high-quality relational care they thrive on. This dissonance between the care they wanted to provide and the reality of the, at times, perceived dangerous staffing, created the impression of significant moral injury and distress for some respondents.

\section{"I just can't do it anymore" : the tipping point of collapse}

As an end to lockdown restrictions was declared on July $19^{\text {th }} 2021$, residents of England saw the widespread resumption of something close to normal activities. Contrary to this, within maternity services, many staff and Heads of Service interviewed were facing the most challenging times of their career. On several occasions in late 2021, our interviews were rescheduled due to Trusts being in critical incident mode. Staff reported being unsure of their future or their roles and described experiencing compassion fatigue towards both their colleagues and those in their care. 'Exhausted', 'broken' and 'unable to carry on' or similar terms were used by a majority of professional participants. Just a small number reporting feeling minimally affected - in one example, the seniority of their role conferred a greater autonomy over workload and the individual felt that control was essential to their management of wellbeing. Many senior midwives, obstetricians and Heads of Service expressed concern about rising incidence of burnout and breakdown leading to an exodus of staff whom they could not replace, placing particular emphasis on what was described by a senior obstetrician as "the biggest midwifery crisis of all time." 


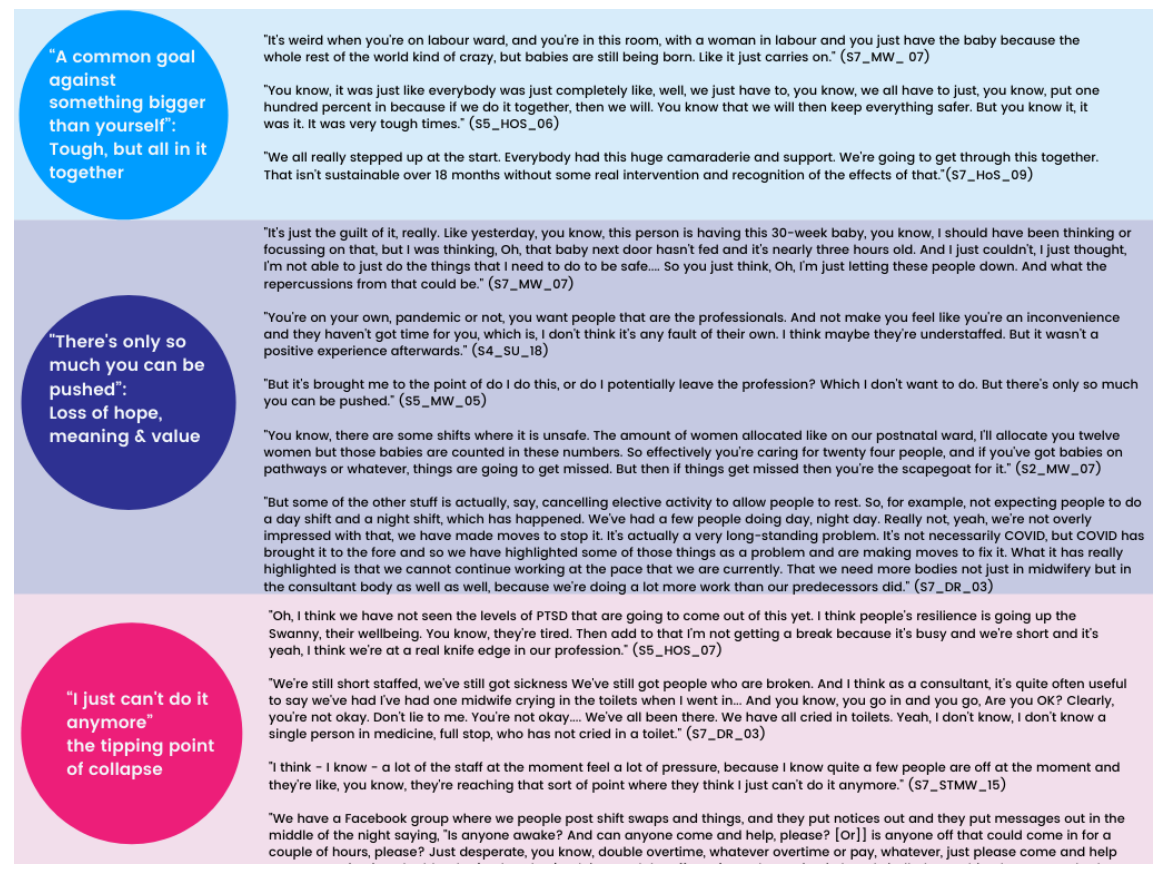

Figure 2: Participant ID: These have been anonymised to protect participants. $\mathrm{MW}=$ midwife $\mathrm{DR}=$ doctor $\quad \mathrm{SU}=$ service user $\mathrm{HoS}=$ head of service $\mathrm{STMW}=$ student midwife

\section{Comment}

Our findings show that interviewees overwhelmingly felt that the COVID-19 pandemic has magnified the existing and escalating staffing crisis, impacting on the ability to provide safe and personalised care. Even pre-pandemic, there were growing concerns about the wellbeing of maternity staff, with high levels of anxiety, depression and burnout noted amongst UK obstetricians and midwives $(7,8)$. In line with our findings, recent research demonstrates that being unable to practise the person-centred and autonomous midwifery care they aspired to is often cited as a reason why midwives consider leaving the profession, although lack of autonomy, pay and conditions are also relevant factors $(9,10)$

Maternity professionals are subject to a broad range of occupational factors that can inhibit their ability to be compassionate, resulting in compassion fatigue and inability to provide quality care (11). The problem does not lie within the job itself - providing relational care may in fact protect against burnout (8). The inability to work in ways that are congruent with vocational identity and professional requirements risks psychological and physical safety for women and babies. These stressors result in a culture of self-sacrifice where maximum levels of effort are required to maintain care, with minimal resources (12). They include working long hours/beyond allotted hours without breaks, lack of time, lack of professional autonomy, challenging clinical situations, lack of support, high levels of fear relating to compromised safety and potential retribution, and witnessing the mistreatment and/or traumatic treatment of women whilst feeling unable to intervene $(9,10)$. Many of these pressures may be exacerbated by insufficient staffing (10). In line with this, safe staffing may mitigate the impact of these stressors on psychological wellbeing (9). As these stressors increase and the ability to provide compassionate care decreases, workers may experience a lack of professional accomplishment, emotional exhaustion, cognitive dissonance, and moral distress (12). As a result, they can withdraw from women and their commitment to the profession. This is aptly described as 'fighting a losing battle' (10). 
Our findings demonstrate how COVID-19 has exposed fundamental flaws in a system where organisational priorities and professional values come into daily conflict. It is increasingly evident that the NHS functions as a result of the goodwill of its workers, which is rapidly running out. It appears from our data that the coping mechanisms (for example, peer support within a well-established, sustainable team, and the freedom to offer compassionate, relational care) that made this working environment manageable are breaking down, leading to burnout and a critical tipping point of collapse. There is growing anecdotal evidence that an increasing number of those working in maternity services are questioning why they come to work every day and are looking elsewhere for employment that is associated with less fear, and less potential for psychological harm.

Our data indicate the extent to which these underlying fault lines have been magnified by the pandemic, to the point where they have become unsustainable. This unique opportunity for a post-pandemic rebuild of the maternity services needs to start by examining how such long-standing flaws in maternity service design and delivery can be built out, to avoid a similar collapse of wellbeing and morale under future organisation-wide stressors. Understanding the protective factors which sustain psychological and physical wellbeing amongst maternity staff, such as flexible and autonomous practice, the ability to provide person-centred, relational care, and strong peer networks, should underpin future efforts to train, recruit and retain this valuable professional workforce.

(1757 words)

Authorship as per BJOG Criteria:

$\mathrm{SC}$ was a research site lead, coordinated and undertook interviews, transcribed data, contributed to the analysis framework, analysed interviews, interpreted analysis, contributed to the concept for paper, coordinated and took responsibility for drafting and revising the paper, approved the final version, and agrees to be accountable for all aspects of the work.

GM transcribed data, contributed to the analysis framework, analysed interviews, interpreted analysis, contributed to the concept for paper, contributed to writing the paper, supported the coordination of drafting and revising the paper, approved the final version, and agrees to be accountable for all aspects of the work.

SD was responsible for study conception, design, ethics, and transcribed data, contributed to the analysis framework, analysed interviews, interpreted analysis, contributed to the concept for paper, contributed to writing the paper, supported the coordination of drafting and revising the paper, approved the final version, and agrees to be accountable for all aspects of the work.

JC contributed to the analysis framework, analysed interviews, interpreted analysis, contributed to the concept for paper, contributed to writing the paper, supported the coordination of drafting and revising the paper, approved the final version, and agrees to be accountable for all aspects of the work.

AS contributed to the analysis framework, analysed interviews, interpreted analysis, contributed to the concept for paper, contributed to writing the paper, supported the coordination of drafting and revising the paper, approved the final version, and agrees to be accountable for all aspects of the work.

CK contributed to project design, obtaining ethics approval, coordinating and managing data collection, developing a framework, revising the paper critically for important intellectual content, approved the final version, and agrees to be accountable for all aspects of the work.

DP contributed to data acquisition, analysis of data, developing a framework, revising the paper critically for important intellectual content, approved the final version, and agrees to be accountable for all aspects of the work.

CF contributed to data acquisition, developing a framework, revising the paper critically for important intellectual content, approved the final version, and agrees to be accountable for all aspects of the work.

Joanne Cull is funded by a National Institute for Health Research (NIHR) Wellbeing of Women Doctoral Fellowship. The views expressed in this paper are those of the authors and not necessarily those of Wellbeing 
of Women, the NHS, the NIHR or the Department of Health and Social Care

\section{Funding Statement}

This research is funded by the Economic and Social Research Council (ESRC), as part of UK Research and Innovation's rapid response to COVID-19 [grant number ES/V004581/1]. Full details of the main study are available via ResearchRegistry (researchregistry5911) and via UKRI Gateway (https://gtr.ukri.org/projects?ref=ES\%2FV004581\%2F1).

\section{Governance and ethics}

The study received Health Research Authority (HRA) and Research Ethics Committee (REC) approval on 23rd October 2020 (IRAS285693). The application was fast-tracked for approval and assessed as under the scope of the COPI Notice. It was adopted onto the NIHR Portfolio. The study was assessed as not in scope of the urgent public health stream. Individual Trusts made their own priority assessment in accordance with the RESTART Framework or RECOVERY Framework. All interview participants provided informed consent, audio recorded using MS Teams and on an electronic consent form. We have endeavoured to protect participants' anonymity and participating sites by removing identifying information from the data.

\section{Data availability statement}

All relevant interview data will be openly available from the UK Data Service's online data repository ReShare and a DOI provided on paper acceptance.

Acknowledgements:With thanks to Anastasia Topalidou for contributions to conception and design, and project management; to Eleanor Smith for project administration; and to Dr Stephanie Heys for data acquisition. The authors would like to thank the ASPIRE COVID-19 Collaborative Group for their valuable time and input.

\section{References}

1. Curtis P, Ball L, Kirkham M. Why do midwives leave? (Not) being the kind of midwife you want to be. British Journal of Midwifery. 2006;14(1):27-31

2. The Health and Social Care Committee's Expert Panel: Evaluation of the Government's progress against its policy commitments in the area of maternity services in England [Internet]. https://committees.parliament.uk/. 2021 [cited 21 December 2021]. Available from:https://committees.parliament.uk/publications/6560/documents/71747/default/

3. MBRRACE-UK. Term, singleton, intrapartum stillbirth and intrapartum-related neonatal death [Internet]. 2017. Available from:https://www.npeu.ox.ac.uk/assets/downloads/mbrrace-uk/reports/MBRRACEUK\%20Intrapartum\%20Confidential\%20Enquiry\%20Report\%202017\%20-\%20final\%20version.pdf

4. Healthcare Safety Investigation Branch. Intrapartum stillbirth: learning from maternity safety investigations that occurred during the COVID-19 pandemic 1 April to 30 June 2020 [Internet]. 2021. Available from:https://www.hsib.org.uk/investigations-and-reports/intrapartum-stillbirth-during-covid-19/

5. Horsch A, Lalor J, Downe S. Moral and mental health challenges faced by maternity staff during the COVID-19 pandemic. Psychological Trauma: Theory, Research, Practice, and Policy. 2020;12(S1):S141S142.

6. Braun V, Clarke V. Conceptual and design thinking for thematic analysis. Qualitative Psychology. 2021;.

7. Bourne T, Shah H, Falconieri N, Timmerman D, Lees C, Wright A et al. Burnout, well-being and defensive medical practice among obstetricians and gynaecologists in the UK: cross-sectional survey study. BMJ Open. 2019;9(11):e030968.

8. Hunter B, Fenwick J, Sidebotham M, Henley J. Midwives in the United Kingdom: Levels of burnout, depression, anxiety and stress and associated predictors. Midwifery. 2019;79:102526. 
9. Cramer E, Hunter B. Relationships between working conditions and emotional wellbeing in midwives. Women and Birth. 2019;32(6):521-532.

10. Geraghty S, Speelman C, Bayes S. Fighting a losing battle: Midwives experiences of workplace stress. Women and Birth. 2019;32(3):e297-e306.

11. Sinclair S, Raffin-Bouchal S, Venturato L, Mijovic-Kondejewski J, Smith-MacDonald L. Compassion fatigue: A meta-narrative review of the healthcare literature. International Journal of Nursing Studies. 2017;69:9-24.

12. Patterson J, Hollins Martin C, Karatzias T. Disempowered midwives and traumatised women: Exploring the parallel processes of care provider interaction that contribute to women developing Post Traumatic Stress Disorder (PTSD) post childbirth. Midwifery. 2019;76:21-35.

\section{Hosted file}

Table 1 Quotes for inclusion in main body of paper "There's only so much you can be pushed" A commentar available at https://authorea.com/users/457314/articles/558204--there-s-only-so-much-youcan-be-pushed-a-commentary-on-the-magnification-of-the-maternity-staffing-crisis-by-the2020-21-covid-19-pandemic

\section{Hosted file}

Appendix A1 "There's only so much you can be pushed" BJOG-22-0125.docx available at https://authorea.com/users/457314/articles/558204--there-s-only-so-much-you-can-bepushed-a-commentary-on-the-magnification-of-the-maternity-staffing-crisis-by-the-202021-covid-19-pandemic

\section{Hosted file}

Table S1_SupplementaryInfo_Quotes Table_BJOG-22-0125.docx available at https://authorea.com/ users/457314/articles/558204--there-s-only-so-much-you-can-be-pushed-a-commentary-onthe-magnification-of-the-maternity-staffing-crisis-by-the-2020-21-covid-19-pandemic 